\title{
Risk management of hazardous material transportation
}

\author{
J. Augutis, E. Uspuras \& V. Matuzas \\ Lithuanian Energy Institute, Lithuania
}

\begin{abstract}
In recent years on Lithuanian roads a considerable amount of hazardous materials have been transported, especially oil products. However, there is no common methodology which could assess the risk of such transportation. Lithuania has accepted directives, norms and other acts of law related to risk and hazard assessment and prevention that are valid in the European Union. In relation to this situation, hazard assessment and analysis has even greater significance. The novelty of the work is associated with employing the Markov process to describe a hazard distribution mechanism and to determine a limited hazard distribution in the nodes of networks.
\end{abstract}

Keywords: Hazard, risk, risk sources, risk distribution, Markov process.

\section{Introduction}

Hazard identification and assessment are rather complicated tasks, which have received attention in the literature (Adams [1]).

Risk of any activity or process is often defined as a set made of $n$ pairs of frequency of hazardous events and their outcomes. Sometimes these values are multiplied. Hazard measurement is less clearly defined and here such qualitative evaluations as high hazard level, medium hazard level, low hazard level, etc. are used. In certain cases, quantitative expressions are also used.

During transportation of hazardous materials or at the outspread of communicable diseases, etc. hazard is divided, moved from one place to the other, distributed among various structures. In the literature, much attention is devoted to the investigation of various hazard distribution mechanisms. One of the most widely explored among these is the distribution of different pollution 
materials in the atmosphere, water and soil, spread of communicable diseases among people, animals and plants (Lefevre and Picard [2]) and shipping hazardous materials with different means of transportation (Purdy [3]). Latter works provide detailed analyses of distribution mechanisms, speed, process duration, etc. of hazardous materials and diseases.

It is also obvious that together with the improvement of means of transportation and the increase in the quantity and size of the loads, the assessment of hazard distribution becomes more prominent in the systems of transportation. It has to be noted that the majority of the scientific research articles and works on the hazard distribution assessment in the network systems has been made during the last several decades and this topic is still under active investigation.

The main aim of the paper is the analysis of hazard distribution in the network systems. In the context of the analysis, hazard is understood as the amount of hazardous materials, disease concentration, etc. Hazard can be distributed through the channels of various networks and concentrated in the nodes of the networks. Hazard transmission through the channels that connect network nodes can take place in many ways: for example, hazard can be transmitted to a single or to several nodes, as an undivided value or divided into parts. Each node can also have certain protection or immunity against hazard, which blocks its transmission or diminishes it.

The paper has a purpose to present the analysis and mathematical model of hazard distribution in the nodes of the network.

\section{Definitions of hazards, transfers and other concepts}

As it was already mentioned, hazard in this paper is equalled to such numerical values as the quantity of hazardous materials, the intensity of informational transfer, etc. Hazard will be noted as $H$. We will now define several terms that will be used in the paper:

Hazard source. It is one of the network nodes in which hazard can arise or occur. Point source of hazard. It is a source of hazard in which hazard occurs only once. Infinitive source of hazard. It is a source of hazard in which hazard arises periodically, for an infinitive number of times.

Additive hazard. It is a sort of hazard, when hazards in the nodes of the network can be added to or a part of hazard moved to the other nodes. The examples of the additive hazard are: collection of hazardous materials, transport intensity, etc. Non-additive hazard. It is a sort of hazard when the sum of hazards is equal to the maximum of those hazards: $H_{1}+H_{2}=\max \left\{H_{1} ; H_{2}\right\}$.

Transfer intensity coefficient between the network nodes. It is a coefficient $q_{i, j}$ that marks the part of the hazard in the node $i$ that will be transmitted to the node $j$. It is clear that $\sum_{j=1}^{N} q_{i, j} \leq 1$, 
here $N-$ a number of network nodes. The transfer intensity to the node $j$ is $\breve{q}_{j}=\sum_{\substack{i=1 \\ i \neq j}}^{N} q_{i, j}$ and from the node $j$ is $\widehat{q}_{j}=\sum_{\substack{k=1 \\ k \neq j}}^{N} q_{j, k}$.

Network. It is system defined as an oriented graph, in which hazard from one node can be transmitted only to one node during a cycle.

Network node immunity. It is a coefficient $I_{j}$ that marks which part of the hazard is transmitted to the node $j\left(0 \leq I_{j} \leq 1\right)$. Node immunity can be created by the security systems, ant-virus computer software, etc.

Transfer probability. It is a probability $p_{i, j}$, that during one cycle hazard from the node $i$ will be transmitted to the node $j$. Transfer probability has the following feature: $\sum_{j=1}^{n} p_{i, j}=1$.

Hazard transfer cycle. Hazard transfer in the network from one node to the other is regarded as one hazard transfer cycle.

Hazard in network nodes after $k$ cycles. Hazard that is accumulated in the node $i$ after $k$ cycles, will be marked as $H_{i}(k)$.

Marginal hazard in the network nodes. Marginal hazard in the $i$ node is a steady hazard after an infinitive number of cycles. $H_{i}=\lim _{n \rightarrow \infty} H_{i}(n)$.

\section{Additive hazard distribution in the network}

The distribution of hazard that can be divided or added in the network nodes will be analysed. Two hazard distribution methods will be analysed separately.

In the first case it will be assumed that hazard can be transferred from every node only to one of the possible nodes, while in the second case, let us allow the hazard spreading though the entire network.

\subsection{Hazard distribution in Markov chains}

Let us suppose that we have a network with $N$ nodes. Hazard from the node $i$ can be transferred only to one node $j$, which is selected according to transfer probability $P_{i j}$. Thus, during each cycle, hazard can occur in only one network node. In the paper an assumption will be made that transfer probabilities have Markov properties. Thus, if the hazard that exists in node $i$ after $n$ cycles will be marked as $X(n)$, so

$$
\begin{aligned}
& P_{i j}=P(X(n)=j \mid X(n-1)=i)= \\
& =P\left(X(n)=j \mid X(1)=i_{1} ; X(2)=i_{2} ; \ldots ; X(n-1)=i_{n-1}\right)
\end{aligned}
$$


This way the process $X(n)$ will be Markov chain with finite set of the states $\{1 ; 2 ; \ldots ; N\}$. The homogeneous Markov chain should also be discussed since $P_{i j}$ is not dependent on $n$. Let us mark hazard occurrence probability in the $i$ node after $n$ cycles $\pi_{i}(n)$. It is clear that $\sum_{i=1}^{N} \pi_{i}(n)=1$.

Now it can be returned to the hazard calculation in each node after $n$ cycles. Naturally, it is possible to determine only average hazard $\bar{H}_{i}(n)$ in each node since hazard after $n$ steps is a random value. If we would also make an assumption that all the network line flows are equal to 1 , the following would be obtained:

$$
\bar{H}_{i}(n)=\frac{1}{n} \sum_{i=1}^{n} H \pi_{i}(k)=\frac{H}{n} \sum_{k=1}^{n} \pi_{i}(k)
$$

Here $H$ - the hazard that has occurred in one of the network nodes during zero step, i.e., we hold that this node is a point source of the hazard.

From the theory of the Markov chains we know that state probabilities after $n$ cycles are described using recursive formulas

$$
\left[\pi_{1}(1), \pi_{2}(1), \ldots, \pi_{N}(1)\right]=\left[\pi_{1}(0), \pi_{2}(0), \ldots, \pi_{N}(0)\right] \cdot\left[P_{i j}\right]
$$

Or, to put it simpler,

$$
\vec{\pi}(1)=\vec{\pi}(0) P
$$

here $P=\left[P_{i j}\right]$ - transfer probability matrix and $\vec{\pi}(0)=[1,0,0, \ldots, 0]$, if we make an assumption that the point source of the hazard is located in the first node.

Then it follows:

$$
\vec{\pi}(2)=\vec{\pi}(1) P=(\vec{\pi}(0) P) P=\vec{\pi}(0) P^{2}, i, j=1,2, \ldots, N
$$

Given that $\vec{\pi}(0)=[1,0,0, \ldots, 0]$, we receive:

$$
\vec{\pi}(n)=\vec{\pi}(n-1) P=\vec{\pi}(0) P^{n-1}=\left[P_{1,1}^{(n-1)}, P_{1,2}^{(n-1)}, \ldots, P_{1, N}^{(n-1)}\right]
$$

Thus, we can calculate the average hazard in the node $i$ after $n$ cycles $\bar{H}_{i}(n)$ recursively, using the following formula:

$$
\bar{H}_{i}(n)=\frac{H}{n} \sum_{k=1}^{n} P_{1 i}^{(k-1)}
$$

here $P_{1 i}^{(k-1)}$ is the $i$ element of the first line of matrix $P^{k-1}$.

According to the eqn. (2), it is not difficult to prove the theorem of the marginal distribution of the hazard average, when $n$ converge to infinity. 
If the Markov chain with $N$ states and transfer probability matrix $P=\left\lfloor P_{i j}\right\rfloor$ is ergodic, i.e., $\lim _{n \rightarrow \infty} \pi_{i}(n)=\pi_{i}, i=1,2, \ldots, N$, so marginal hazard average values in all the nodes of the network also exist.

When in the eqn. (2) we reach the limit when $n$ converge to infinity we get:

$$
\lim _{n \rightarrow \infty} \bar{H}_{i}(n)=\lim _{n \rightarrow \infty} \frac{H}{n} \sum_{k=1}^{n} \pi_{i}(k)
$$

As $\lim _{k \rightarrow \infty} \pi_{i}(k)=\pi_{i}$, so there is vanishing function $\varepsilon(k)$ which is $\pi_{i}(k)=\pi_{i}+\varepsilon_{k}(i)$, where $\lim _{n \rightarrow \infty} \varepsilon_{k}(i)=0, k=1,2, \ldots$. Then

$$
\begin{aligned}
& \lim _{n \rightarrow \infty} \bar{H}_{i}(n)=\lim _{n \rightarrow \infty} \frac{H}{n} \sum_{k=1}^{n}\left(\pi_{i}+\varepsilon_{k}(i)\right)= \\
& =\lim _{n \rightarrow \infty}\left(\frac{H}{n} \pi_{i}\right) n+\lim _{n \rightarrow \infty} \frac{H}{n} \sum_{k=1}^{n} \varepsilon_{k}(i)
\end{aligned}
$$

Let us select $\varepsilon_{(i)}^{(n)}=\max _{1 \leq k \leq n}\left\{\varepsilon_{1}(i) ; \varepsilon_{2}(i) ; \ldots ; \varepsilon_{n}(i)\right\}$. Then:

$$
0 \leq \lim _{n \rightarrow \infty} \frac{H}{n} \sum_{k=1}^{n}\left|\varepsilon_{k}(i)\right| \leq \lim _{n \rightarrow \infty} \frac{H}{n} \cdot n \varepsilon^{(n)}(i)=0
$$

Let $\lim _{n \rightarrow \infty} \frac{H}{n} \sum_{k=1}^{n} \varepsilon_{k}(i)=0$, and, therefore, $\bar{H}_{i}=H \pi_{i}$.

Thus, the marginal average hazard exists in every node, besides, it is equal to the product of the initial $H$ and the marginal node probability.

\subsection{The distribution of the additive hazard in the network nodes during the transitional period}

In this section, a hazard which is characterized by a value that can be summed or divided as a real number will be analysed. The examples of such hazard are concentrations of significant amounts of hazardous materials, concentration of pollution materials, the amount of water that rises in the reservoir, etc. For calculation of hazard in each network node during the transitional period, systems of equation were made, conditions for marginal hazard existence were specified and for the calculation of marginal hazards in the network systems, systems of equation were formed. 
We analyse a network system in which hazard from each node can be transferred to other nodes during one cycle, by dividing hazard $H_{i}(n)$ of the $i$ node in proportion to the flows $q_{i j}$, when $j=1,2, \ldots, N$ and $\sum_{j=1}^{N} q_{i j} \leq 1$.

First of all, let us assume that one network node, for example, the first one, is a point source of the additive hazard, in which hazard $H_{1}(0)$ occurs. Thus, at the zero step we have the following hazard distribution in the nodes:

$$
\vec{H}(0)=\left[\begin{array}{llll}
H_{1}(0) & 0 & \ldots & 0
\end{array}\right]
$$

During the following cycles, hazard modification will occur in each node. From that node hazard will be transferred to other nodes by flows $q_{i j}$. The total transfer will be:

$$
H_{i}(n)\left(q_{i 1}+q_{i 2} \ldots+q_{i, i-1}+q_{i, i+1}+\ldots+q_{i, N}\right)=H_{i}(n) \widehat{q}_{i}
$$

part of hazard. In the node $i$ it will remain

$$
H_{i}(n)-H_{i} \widehat{q}_{i}=H_{i}(n) q_{i i}
$$

part of hazard. The hazard $\sum_{j=1}^{N} H_{j i} q_{q 1}$ will be respectively transferred from other nodes to the node $i$. Thus, after $n$ cycles, we will have the following hazard in the node $i$ :

$$
H_{i}(n+1)=H_{1}(n) q_{i 1}+H_{2}(n) q_{i 2}+\ldots+H_{N}(n) q_{i N} \text {, where } i=1,2, \ldots, N \text {. }
$$

After defining network transfer matrix $Q=\left\lfloor q_{i j}\right\rfloor$, we can write the system of equations in the form of matrix:

$$
\vec{H}(n+1)=\vec{H}(n) \cdot Q
$$

Thus, we have received hazard distribution in the iterative process. As the process is stationary, i.e. matrix $Q$ is not dependent on the number of cycles $n$, so irrespective of the initial hazard distribution, this process converges only when all matrix $Q$ own values will be less than one. This is as well the obligatory and sufficient condition for the marginal distribution of the additive hazard in the network systems.

It is easy to ascertain that if the sum of the elements of the lines in square matrix $A=\left\lfloor a_{i j}\right\rfloor$ is $\sum_{j=1}^{N} a_{i j}=1$, where $a_{i j}<1$, then the sum of the elements of the lines in matrix $A^{n}$ is also equal to 1 , and its elements are $a_{i j}(n)<1$. 
The iterative process of hazard distribution $\vec{H}(n)$ converges when the number of cycles is $n \rightarrow \infty$ and it is not dependent on the initial hazard distribution, if all the flows are $0<q_{i j}<1$.

It is important to analyse risk distribution after the certain number of iterations.

From the eqn. (14) follows that:

$$
\vec{H}(n+1)=\vec{H}(n-1) Q \cdot Q=\vec{H}(n-1) Q^{2}=\ldots=\vec{H}(0) Q^{n+1}
$$

Therefore

$$
\vec{H}(n+1)=\vec{H}(0) Q^{n+1}
$$

This equality allows employing the ideas that are used when proving the ergodic theorems of Markov's chain states.

We shall mark the elements of matrix $Q^{n}$ this way: $q_{i j}(n)$, and $q_{i j}=q_{i j}(1), i, j=1,2, \ldots, N$. We will first notice that

$$
q_{i j}(n)=\sum_{l=1}^{N} q_{i l} q_{l j}(n-1) \geq \min _{1 \leq l \leq N} q_{l j}(n-1) \sum_{l=1}^{N} q_{i l}=\min _{1 \leq l \leq N} q_{l j}(n-1)
$$

because $\sum_{l=1}^{N} q_{i l}=1$. This feature is also correct with $q_{i j}(n)$, for which $q_{i j}(n)=\min _{1 \leq j \leq N} q_{i j}(n-1)$. Thus,

$$
\min _{1 \leq i \leq N} q_{i j}(n) \geq \min _{1 \leq j \leq N} q_{i j}(n-1)
$$

By analogy, it is possible to show that

$$
\max _{1 \leq i \leq N} q_{i j}(n) \leq \max _{1 \leq i \leq N} q_{i j}(n-1)
$$

Let us evaluate $q_{i j}(n)-q_{l j}(n)$, for all $i, j, l=1,2, \ldots, N$. Of course, for any $s<n$ :

$$
\begin{aligned}
& q_{i j}(n)-q_{l j}(n)=\sum_{r=1}^{N} q_{i r}(s) q_{r j}(n-s)-\sum_{r=1}^{N} q_{l r}(s) q_{r j}(n-s)= \\
& =\sum_{r=1}^{N}\left[q_{i r}(s)-q_{r j}(s)\right] q_{r j}(n-s)
\end{aligned}
$$

Positive differences $q_{i r}(s)-q_{l r}(s)$ will be marked as $\beta_{i l}^{(r)}(+)$ and negative ones as $\beta_{i l}^{(r)}(-)$. As 


$$
\sum_{r=1}^{N} q_{i r}(s)=\sum_{r=1}^{N} q_{l r}(s)=1
$$

Thus,

$$
0=\sum_{r=1}^{N}\left[q_{i r}(s)-q_{l r}(s)\right]=\sum_{(r)} \beta_{i l}^{(r)}(+)-\sum_{(r)} \beta_{i l}^{(r)}(-)=0
$$

Let us mark

$$
v_{i j}=\sum_{(r)} \beta_{i l}^{(r)}(+)=\sum_{(r)} \beta_{i l}^{(r)}(-)
$$

As all the $q_{i j}(s)>0$, so

$$
\sum_{(r)} \beta_{i l}^{(r)}(+)<\sum_{r=1}^{N} q_{i r}=1
$$

Therefore, $0 \leq v_{i j}<1$. Let us mark $v=\max _{1 \leq i, j \leq N} v_{i j}$. Then $0 \leq v<1$. It is now clear that:

$$
\begin{aligned}
& \left|q_{i j}^{(n)}-q_{l j}(n)\right|=\left|\sum_{r=1}^{N} \beta_{i l}^{(r)}(+) q_{r j}(n-s)-\sum_{r=1}^{N} \beta_{i l}^{(r)}(-) q_{r j}(n-s)\right| \leq \\
& \leq\left|\max _{1 \leq r \leq N} q_{r j}(n-s) \sum_{r=1}^{N} \beta_{i l}^{(r)}(+)-\min _{1 \leq r \leq N} q_{r j}(n-s) \sum_{r=1}^{N} \beta_{i l}^{(r)}(-)\right| \leq \\
& \leq v\left|\max _{1 \leq r \leq N} q_{r j}(n-s)-\min _{1 \leq r \leq N} q_{r j}(n-s)\right| \leq v \max _{1 \leq i, l \leq N}\left|q_{i j}(n-s)-q_{l j}(n-s)\right|
\end{aligned}
$$

For all $i, l=1,2, \ldots, N$.

Then,

$$
\max _{1 \leq i, l \leq N}\left|q_{i j}(n)-q_{l j}(n)\right| \leq v \max _{1 \leq i, l \leq N}\left|q_{i j}(n)-q_{l j}(n)\right|
$$

After using this recursive inequality for $] \frac{n}{s}[$ of times we get:

$$
\max _{1 \leq i, l \leq N}\left|q_{i j}(n)-q_{l j}(n)\right| \leq v^{\frac{n n}{s}[} \max _{1 \leq i, l \leq N}\left|q_{i j}(n-] \frac{n}{s}[\cdot s)-q_{l j}(n-] \frac{n}{s}[\cdot s)\right|
$$

From previous statements $0<q_{i j}(s)<1$, so $\left|q_{i j}(s)-q_{l j}(s)\right| \leq 1$.

From that we get

$$
\max _{1 \leq i, l \leq N}\left|q_{i j}(n)-q_{l j}(n)\right| \leq v^{] \frac{n}{s}[}
$$


Then $\lim _{n \rightarrow \infty} \max _{1 \leq i, l \leq N}\left|q_{i j}(n)-q_{l j}(n)\right|=0$.

Let us remember the inequalities of this proof eqn. (18) and eqn. (19). We get that flows $\left\{\min _{\leq \leq i \leq N} q_{i j}(n)\right\}$ and $\left\{\max _{1 \leq i \leq N} q_{i j}(n)\right\}$ are monotonous and definite $\left(0<q_{i j}(n)<1\right)$, which means that they have limits:

$$
q^{*}=\lim _{n \rightarrow \infty} \max _{1 \leq i \leq N} q_{i j}(n) \text { and } q^{* *}=\lim _{n \rightarrow \infty} \min _{1 \leq i \leq N} q_{i j}(n) .
$$

From the eqn. (28) we obtain that there is a limit $q_{j}=\lim _{n \rightarrow \infty} q_{i j}=q_{j}^{*}=q_{j}^{* *}$. It is clear that $1=\lim _{n \rightarrow \infty} \sum_{j=1}^{N} q_{i j}(n)=\sum_{j=1}^{N} q_{j}$.

Let us return to the eq. (14). When we come to the limit of $n \rightarrow \infty$, we mark $\lim _{n \rightarrow \infty} Q^{n+1}=\bar{Q}, \lim _{n \rightarrow \infty} \vec{H}(n+1)=\bar{H}$, and get:

$$
\lim _{n \rightarrow \infty} \vec{H}(n+1)=\lim _{n \rightarrow \infty} \vec{H}(0) Q^{n+1}=H(0) \bar{Q}
$$

Or

$$
\vec{H}=\vec{H}(0) \bar{Q}=\left[\begin{array}{lll}
H & 0 & \ldots
\end{array}\right]\left[\begin{array}{cccc}
q_{1} & q_{2} & \ldots & q_{N} \\
q_{1} & q_{2} & \ldots & q_{N} \\
\ldots & \ldots & \ldots & \ldots \\
q_{1} & q_{2} & \ldots & q_{N}
\end{array}\right]
$$

Thus,

$$
\vec{H}=\left[\begin{array}{llll}
H q_{1} & H q_{2} & \ldots & H q_{N}
\end{array}\right]
$$

This distribution does not depend on initial conditions.

\section{The main results and conclusions}

The main aim of the paper is to present the developed hazard distribution mathematical model and its analysis. Here was analysed the mechanism of hazard propagation in network systems in the case of single hazard, evolved in one of the network nodes and in case when hazard arise during each cycle. Few network system cases were analysed. The most important cases are hazard propagation in Markov chains and network systems, where peaks have an immunity or resistance to the hazard characteristic.

\section{References}

[1] Adams, J., Risk, UCL Press Ltd, London, 1995. 
402 Management of Natural Resources, Sustainable Development and Ecological Hazards

[2] Lefevre, C., Picard, P., An epidemic Model with Fatal Risk, Mathematical Biosciences, NY, 117, pp. 127-145, 1993.

[3] Purdy, G., Risk Analysis of the Transportation of Dangerous goods by Road and Rail, Journal of Hazardous Materials, 33, pp. 229-259, 1993. 